\title{
Mujeres en la música experimental y colectivos feministas en estudios sonoros en Brasil
}

Women in Experimental Music and Feminist Groups in Sound Studies in Brazil 


\title{
Mujeres en la música experimental y colectivos feministas en estudios sonoros en Brasil
}

\section{Women in Experimental Music and Feminist Groups in Sound Studies in Brazil}

\author{
Isabel Nogueira ${ }^{1}$ \\ Universidad Federal de Rio Grande do Sul \\ Porto Alegre, Brasil \\ Tania Mello Neiva ${ }^{2}$ \\ Universidad Federal da Paraíba \\ João Pessoa, Brasil
}

Recibido: 23 de agosto de 2017 Aprobado: 24 de octubre de 2017

\begin{abstract}
Resumen
Este trabajo es parte de un proyecto en curso sobre una posible cartografía de las compositoras, productoras y creadoras de música experimental y arte sonoro en Brasil. Tiene por objetivo presentar y discutir, de forma inicial, los procesos de formación e intercambio de las redes de creación sonora experimental de mujeres y colectivos feministas en Brasil, desde el punto de la escucha de las autoras de este artículo. Desde las epistemologías feministas, pensamos los feminismos como un lente para ver el mundo, buscando una forma de pensar que sea disruptiva en su manera de comprender lo que se quiere estudiar. Buscamos presentar festivales, espacios, colectivos, grupos y redes donde se teje y se realimenta la práctica musical feminista y de mujeres en Brasil, para luego traer un listado inicial de artistas.

Palabras clave: estudios del sonido, música y género, música experimental, colectivos feministas, compositoras

1 Profesora titular de la Universidad Federal de Rio Grande del Sur, Brasil. Doctora en História y Ciencias Musicales por la Universidad Autonoma de Madrid, España. Correo electrónico: isabel.isabelnogueira@gmail.com

2 Doctoranda en Musicología por Universidad Federal de Paraíba (UFPB).Master en música por la Universidad Estatal de Campinas (UNICAMP). Correo electrónico: taniamelloneiva@gmail.com
\end{abstract}

ESCENA. Revista de las artes, 2018, Vol. 78, Núm. 1 (julio-diciembre), pp. 98-124. ISSN 2215-4906 


\begin{abstract}
This work is part of an ongoing project on a possible cartography of the composers, producers and creators of experimental music and sound art in Brazil, and aims to present and discuss in an initial way the processes of formation and exchange of networks in experimental sound creation of women and feminist groups in Brazil, from the point of listening of the authors of this article. From feminist epistemologies, we think of feminisms as a lens to see the world, looking for a way of thinking that is disruptive in its way of understanding what we want to study. We aim to present festivals, spaces, groups, groups and networks where the feminist and women's music practice in Brazil is woven and fed, and then bring an initial list of artists.
\end{abstract}

Keywords: sound studios, music and genre, experimental music, feminist collectives, women composers 


\section{Introducción}

Este trabajo es parte de un proyecto en curso sobre una posible cartografía de las compositoras, productoras y creadoras de música experimental y arte sonoro en Brasil. Tiene por objetivo presentar y discutir, de forma inicial, los procesos de formación e intercambio de las redes de creación sonora experimental de mujeres y colectivos feministas en Brasil, desde el punto de la escucha de las autoras de este artículo. Utilizamos la metáfora de los puntos de escucha en referencia a Travassos: "substituindo a metaforização do conhecimento pelo olho e pela visão -quero indicar que a posição do ouvinte (num campo profissional, num eixo geracional, etc.) condiciona sua audição [sustituyendo la metaforización del conocimiento por el ojo y la visión-quiero indicar que la posición del oyente (en un campo profesional, en un eje generacional, etc.) condiciona su audición]" (Travassos, 2005, p. 94).

Para nosotras, es importante demarcar el lugar desde donde hablamos y la forma como pensamos construir nuestra narrativa: desde las epistemologías feministas y del pensamiento decolonial. Para deconstruir la idea de narrativas únicas, estamos de acuerdo con la forma de pensar de la investigación cualitativa, la cual entiende que los investigadores construyen sus datos y elaboran su razonamiento con base en esos datos recolectados e interpretan esos mismos datos en sus lecturas. De hecho, desde las epistemologías feministas, concebimos el feminismo como un lente para ver el mundo, entendiéndolo, no solo como una vía para incluir a las mujeres a fin de compensar la subordinación histórica a la que han estado sometidas, sino asumiéndolo como un pensamiento disruptivo en la comprensión de este sujeto a estudiar: las mujeres.

El feminismo se configura como una epistemología disruptiva puesto que cuestiona las invisibilidades, silenciamientos y feminicidios epistémicos operados contra las mujeres, trayendo, por medio de la no repetición de los patrones patriarcales, el cuestionamiento respecto a la forma como el género opera como marcador político, ético y estético en todos los campos del conocimiento, en donde incluimos la música. Hablando de las epistemologías feministas, entendemos que no se puede pensar en un feminismo único, sino, más bien, en feminismos, una vez que entendemos que las mujeres y los colectivos que estas forman son múltiples como son sus experiencias. En nuestro caso, somos dos mujeres de clase media, originarias del sur y del sureste de Brasil, con formación superior en música y vinculación académica (como profesora y como doctoranda), performers, compositoras e improvisadoras, madres y participantes de colectivos y activismos feministas. Pensamos estructurar este artículo presentando festivales, espacios, colectivos, grupos y redes donde se teje y se realimenta la práctica musical feminista y de mujeres, para luego, traer un listado de artistas, con sus enlaces de referencia.

ESCENA. Revista de las artes, 2018, Vol. 78, Núm. 1 (julio-diciembre), pp. 98-124. ISSN 2215-4906 
Antes de seguir adelante, vale decir que es un reto enorme hablar de un país con las dimensiones continentales que tiene Brasil y con un contexto tan desigual. Además de desigualdades regionales, las dimensiones del país hacen que se establezcan nichos y redes específicas de contactos. Por eso, decimos que estas reflexiones que traemos aquí son, y no podrían dejar de ser, frutos de nuestras vivencias, concepciones y contextos personales, aunque en este momento hagamos el esfuerzo para mirar la totalidad del campo.

También, las narrativas con respecto a la música en el país, se establecen desde la idea de que lo que sucede en Rio de Janeiro y Sao Paulo; es lo que cuenta para la "historia oficial". Mientras que, lo que pasa en otras partes del país, ajenas a esas provincias, es leído como "historias regionales". De esa forma, se han establecido narrativas que son hegemónicas y colonializantes en sus conceptos, puesto que consideran las manifestaciones musicales como dependientes de los modelos de esas provincias. Igualmente, hay que decir que la relación de Brasil con los países americanos de habla hispana no es, exactamente, una relación de proximidad y conocimiento estrecho. Es más fácil, por ejemplo, que un curso de música en nivel de posgrado en Brasil presente una bibliografía completamente en inglés, a que incluyan uno o dos títulos, por lo menos, en español, lo que caracteriza otra expresión de la colonialidad de saberes. El intercambio docente o los países en los que se busca formación internacional suelen ser, en la mayoría de los casos, los de América del Norte o de Europa, con raras excepciones.

Sin embargo, por pensar que los relatos feministas deben constituirse de múltiples miradas, decidimos dejar claro el lugar desde donde hablamos para que se pueda pensar en narrativas que no sean únicas. Es decir, para que sea posible decolonizar los saberes, como propone Chandra Mohanty (1984) al denunciar posturas y discursos colonizantes de teoricas feministas del Norte en relación a las mujeres del "Sur Global" al generalizarlas como oprimidas. Aquí, cuando hablamos de nuestro lugar de habla y de escucha, asumimos nuestra responsabilidad como proponentes de narrativas y posibilidades de miradas, en relación a un contexto, sabiendo que nuestra proposición es también resultado del contexto en el que nos formamos. Entendemos que el primer paso para la construcción no autoritaria o colonizante de narrativas es asumirlas como posibilidades conjeturales. El tema de este artículo presenta, por lo pronto, tres problemas, más que soluciones y de ellos buscaremos ocuparnos uno a uno, para luego entrelazarlos. Una breve discusión de lo que es música experimental, luego los colectivos feministas y, seguidamente, una aproximación a las redes y cómo se estructuran. Para, finalmente, presentar un listado de compositoras/ creadoras/productoras y sus referencias. 


\section{El campo de la música experimental}

El tema de qué es y lo que significa la música experimental se presenta, desde luego, como un problema al cual tenemos que acercarnos buscando comprender sus perspectivas. El término ha sido y, sigue siendo, debatido desde distintos puntos. Para este trabajo traemos algunos conceptos que dialogan con las producciones de los colectivos, iniciativas y artistas que tratamos aquí. Empezamos con la definición de Lílian Campesato (2015) que, después de considerar las aproximaciones y diferencias entre experimento (asociado a las producciones más enfocadas en la comprobación de una tesis, como en el caso de las ciencias biológicas, físicas y exactas) y experimentalismo (asociado a la ruptura con normas rígidas preestabelecidas), concluye:

Experimentar significa testar pela ação aquilo que está ao nosso alcance. O experimentalismo pressupõe colocar em questão o que já é conhecido e é um dos processos que podem levar a um radicalismo (transformação profunda) ou a uma marginalização (fora do centro). Portanto, a atitude experimental é questionadora e leva a conclusões que confrontam o que já é estabelecido pelos hábitos, pelas crenças ou por uma certa inércia [Experimentar significa probar por la acción aquello que está a nuestro alcance. El experimentalismo presupone poner en cuestión lo que ya es conocido y es uno de los procesos que pueden llevar a un radicalismo (transformación profunda) o a una marginalización (fuera del centro), por lo tanto, la actitud experimental es cuestionadora y lleva a conclusiones que confrontan lo que ya está establecido por los hábitos, por las creencias o por una cuestión de inercia] (Campesato, 2015, p. 62).

Sobre la definición, lazzetta (2014) apunta que:

o experimentalismo tem sido frequentemente associado a práticas de caráter transgressor, em que comportamentos, modelos e delimitações bem estabelecidos são colocados em questão. Experimental, significa nesse caso, uma atitude crítica em relação ao que está consolidado e é aceito como referência artística, forçando uma abertura para incorporação de elementos relativamente estranhos a um determinado campo na arte. Na música experimental, o foco na composição se transporta para a performance e a separação entre criação e recepção é atenuada uma vez que o público é chamado a ter um papel mais atuante nos processos de geração e fruição da obra. O traço de transgressão ou subversão torna-se o elemento essencial do experimentalismo [el experimentalismo ha sido frecuentemente asociado a prácticas de carácter transgresor, en la que comportamientos, modelos y delimitaciones bien establecidos son puestos en cuestión. Experimental, significa, en ese caso, una actitud crítica hacia lo que está consolidado y aceptado como referencia artística, forzando una apertura para la incorporación de elementos relativamente extraños a un determinado campo del arte. En la música experimental, el foco en la composición se transporta hacia la performance, y la separación entre creación y recepción es atenuada una vez que el público está llamado a tener un papel más activo en los procesos de generación y fruición de la obra. El rasgo de transgresión o subversión se convierte en un elemento esencial del experimentalismo] (pp. 4-5).

ESCENA. Revista de las artes, 2018, Vol. 78, Núm. 1 (julio-diciembre), pp. 98-124. ISSN 2215-4906 
Por su parte, Henrique Iwao (2012):

Pode-se pensar a música experimental como uma prática que prioriza novas concepções do musical pautadas na experimentação formal. Os músicos praticantes são incentivados a conceber música de um modo altamente pessoal e tentar criar rupturas com os discursos musicais estabelecidos. Como advogaria a criação de novas formas de fazer, a música experimental seria transversal aos vários gêneros musicais, podendo servir-se de características e restrições convencionadas destes, mas não se fixando em nenhum, esquivando-se assim de configurações mais codificadas. Nesse sentido, sinteticamente, poderse-ia pensar a música experimental como um anti gênero. Ficaria também assim impressa a vontade de não fixação desses modos como códigos. (p. 67) [Se puede pensar la música experimental como una práctica que prioriza la creación de nuevas concepciones de lo musical, pautadas en la experimentación formal. Se anima a los músicos practicantes a diseñar música de un modo altamente personal e intentar crear rupturas con los discursos musicales establecidos. Como abogaría por la creación de nuevas formas de hacer, la música experimental sería transversal a los diversos géneros musicales, pudiendo servirse de características y restricciones convencionales de éstos, pero no fijándose en ninguno, se esquivándo así de configuraciones más codificadas. En ese sentido, sintéticamente, se podría pensar en la música experimental como un antigénero] (p. 67).

Entonces, pensando la música experimental como un campo, traemos el concepto de Pierre Bourdieu $(2003,2004)$ que dice que campo es un espacio social de disputa política y económica por mayor prestigio. Las sociedades se dividen en infinitos campos, los cuales también se subdividen. Dentro de cada campo hay reglas que establecen tanto las normas dominantes (incluyendo personas -agentes-, instituciones y creencias), como las que se someten. De manera general, hay disputa por la ocupación del lugar de dominador, sin embargo, las reglas, las instituciones y las relaciones establecidas entre los y las agentes; así como entre los y las agentes y las instituciones, funcionan de forma circular, lo que hace difícil la ruptura con el esquema de producción, reproducción y consagración establecido en el campo en cuestión. Para Bourdieu, la comprensión de una determinada situación social se da por la teoría de las prácticas, del habitus, en la cual la práctica del o de la agente social es considerada en función de las relaciones que determinan la estructuración de la sociedad. El campo es un "espacio social" competitivo entre agentes y habitus. El individuo que ha internalizado en sí una serie de valores y prácticas determinadas por el habitus, está inserto en ciertas situaciones sociales que concuerdan con el habitus en el que se encuentra. Por lo tanto, esto hace difícil el reconocimiento de este como construcción social y, en consecuencia, difícil su ruptura.

En otras palabras: la sociedad está compuesta por infinitos campos, determinados por sus agentes y habitus específicos y por la disputa de poder entre ellos. Dentro de cada 
campo, las relaciones se establecen como dominantes y dominadas, esa relación está determinada por el mismo habitus. Los agentes también se dividen entre los dominantes y los dominados, los cuales aspiran a alcanzar el estatus de dominante del campo, configurando un espacio de luchas internas. La función social ocupada por el (la) agente es reproducida sistemáticamente (a causa de la internalización y naturalización de determinado habitus) y reproduce los procesos de producción y consagración de las reglas del campo en cuestión, propiciando el mantenimiento de una determinada situación. Por eso, dirá Bourdieu, la superación o transformación de una determinada realidad social es tan difícil. Además, se enfatiza la dificultad cuando se pone en relación con la dominación masculina, la cual ocurre en todos los campos sociales que conocemos y es reproducida de forma naturalizada y esencialista (Bourdieu, 2003).

Para nosotras, es importante pensar la música experimental desde el punto de vista de la constitución de un campo definido por las prácticas experimentales en música, tal y como lo plantean lazzetta e Iwao, más que desde su vinculación estética a los compositores de una u otra escuela. Así, entendemos que se configura en un proceso histórico y contingencial. En su tesis de doctorado, recientemente defendida, titulada Prácticas colaborativas en música experimental en Brasil entre 2000 y 2016, Mário Del Nunzio (2017), después de abordar las diferentes posibilidades de conceptualización de lo experimental en la música, en contextos históricos y de diferentes localidades, argumenta que el término música experimental es muy amplio. Su argumentación conduce hacia la idea de que el término se configura como no-restrictivo, siendo incluso, el título de ese trecho de la tesis: "no-género", corroborando la idea de antigénero, propuesta por Iwao (2012). En el caso de las prácticas experimentales en Brasil, en el marco del recorte de propuesto, la idea de música experimental que adopta es la de formación de redes, apoyada, principalmente, en la concepción de Benjamin Piekut.

En diálogo con Del Nunzio, entendemos que el campo de la música experimental brasileña se construye a partir de prácticas, relaciones e intercambios entre compositores y compositoras, productores y productoras, instituciones formales y no formales, iniciativas específicas como los sellos independientes, los blogs, los sitios web, los grupos y las páginas de Facebook, en fin, toda la gama de publicaciones que abordan el campo y se insertan en él; también, los festivales y tantos otros, que conforman una red. Por otra parte, en lo que corresponde a la participación de las mujeres en ese campo, nos encontramos con una multiplicidad de abordajes. Ninguna de ellas delimitadora de un modelo único, tanto de prácticas como de enfoque o denominación y eso, además de lo amplio que es el país, constituye, en definitiva, una dificultad de abordaje que nos exige decisiones específicas. 
En el campo de la música experimental, se podría decir que la forma de denominación no es única. Compositoras, creadoras o productoras: ¿con cuáles palabras se definen las mujeres? Por lo pronto, podemos decir que muchas de las mujeres que trabajan con música experimental no se designan y no desean autodesignarse compositoras. Por distintos motivos, o bien, por no haber realizado estudios formales de composición o por no haber siquiera realizado estudios de música. Esto debido a que la enseñanza universitaria de música sigue unos modelos ya establecidos de trabajo, donde a veces lo disruptivo no tiene lugar; sea porque su interés esté más enfocado hacia las artes visuales, o sea porque su campo de acción no utiliza esta palabra. Ese temor a autodesignarse como compositoras habla, a nuestro modo de ver, de cómo se han estructurado campos opuestos, binarios, que responden a una lógica hegemónica, colonizadora que define subalternos y superiores también en el campo de la música. Existe una variedad de mujeres que trabajan con circuit bending, electrónica, computadoras, artistas sonoras, productoras de la música electrónica de pista, o de sets como DJs. Se puede pensar que, si la academia no se hace atractiva a las expectativas, necesidades y deseos de las mujeres creadoras, ellas busquen otras formas de aprender y actuar.

\section{Estudios sobre música y mujeres en Brasil}

Con relación a la emergencia del tema música y mujeres en Brasil, los primeros estudios datan de finales de la década de 1970, referentes a la compositora Chiquiña Gonzaga. El libro autobiográfico de la compositora Jocy de Oliveira, de 1983, marca una tendencia de los escritos de sí mismas por parte de las compositoras, lo cual tardó mucho por afirmarse como una posibilidad. Por otra parte, Zerbinatti, Nogueira y Pedro citan la existencia de los escritos sobre diversas compositoras, como son los libros de Eli Maria Rocha (1986) y Nilceia Baroncelli (1987), las primeras fuentes dedicadas a reunir ese tipo de información (Zerbinatti, Nogueira y Pedro, 2017, p. 2). En cuanto al movimiento de mujeres en la música en Brasil, citamos el Encuentro Internacional de Mujeres Compositoras, organizado por Maria Helena Rosas Fernandes, que ocurrió de 1993 a 2004 (p. 5).

El Encuentro Internacional de Mujeres Compositoras fue un evento que reunió a compositoras de música erudita de Brasil y de varios países del mundo. En el evento presentaron sus obras a lo largo de tres días, por medio de conferencias, comunicaciones y mesas redondas, además de presentaciones al público en general. Aunque no sea específico del campo de la música experimental, ha sido un evento que reunió un importante número de compositoras y, por tanto, creemos que es importante su referencia. Con la creación de los cursos de posgrado de música en Brasil, a partir del 1987, se intensifica la producción. También, los programas de posgrado en música, los anales de congresos y eventos, las tesis de máster y doctorado, las revistas académicas son formas importantes de visibilidad de esa producción (Zerbinatti, Nogueira y Pedro (2017, p.6). 
Según Zerbinatti, Nogueira y Pedro (2017), en los años 2000 y 2010, especialmente, en diferentes lugares del país, las personas producían -y aún producen- sobre el tema. Una de las temáticas principales de las primeras investigaciones fueron las actividades musicales de mujeres -en su mayoría compositoras- y las prácticas musicales de mujeres en diferentes contextos. La observación de qué trabajos concomitantes existieron está de acuerdo con premisas feministas, que buscan observar el hibridismo de las prácticas y los procesos, así como la inclusión de diferentes lugares de habla y de producción de sentido. Esto para que podamos expandir la comprensión hacia una historia que no se piense única, homogénea y laudatoria, en la que solo se escuchen y reconozcan a pocos personajes (Possas, 2011).

En el 2013, se editó el libro Pesquisa em Música no Brasil (Vol. 3): Estudos de Gênero, Corpo e Música-abordagens metodológicas, por la editora de la ANPPOM, organizado por Isabel Nogueira y Susan Campos Fonseca. El libro ha sido dedicado a la memoria de Maria Ignez Cruz Mello, pianista, compositora, antropóloga y etnomusicóloga, una de las primeras investigadoras sobre música y género en Brasil. Participaron en el libro Susan Campos Fonseca; Teresa Cascudo y Miguel Angel Aguilar Rancel; Maria Palácios; Talitha Couto Moreira; Catarina Domenici; Laila Rosa y Grupo Feminaria Musical; Fabiane Luckow; Isabel Nogueira; Francisca Michelon y Fábio Vergara Cerqueira; Rafael Noleto; Vanda Freire y Angela Portela; Mónica Vermes; Marcia Taborda; Vania Muller, y Rodrigo Cantos Savelli Gomes. En los anexos del libro, tres traducciones de Carlos Palombini; una nueva publicación del verbete "Música lésbica y gay" de Philip Brett y Elizabeth Wood, publicado anteriormente por la Revista Electrónica de Musicología; una entrevista con Elizabeth Wood, y el prefacio de Wayne Koestenbaum para Queering the pitch de Philip Brett, Elizabeth Wood y Gary Thomas. Se hace significativa esa referencia por el hecho de haber sido producida por la Asociación Nacional de Investigación y Posgrado en Música de Brasil y por congregar muchos investigadores e investigadoras.

A continuación, buscamos referir proyectos que están dentro de las universidades, pero que también forman redes más allá de ellas, según los conceptos de que las epistemologías feministas actúan pensando teoría, práctica, reflexión y activismos como elementos interrelacionados.

\section{Redes, colectivos, grupos, blogs y proyectos}

Las redes son sinónimo de intercambios. Sin embargo, no todas las redes se desarrollan de forma lineal o cuentan con integrantes que tienen perspectivas similares, vivencias o conceptos uniformes de lo que es el feminismo o el objetivo de la red. Por lo anterior, una de las proposiciones clave de las epistemologías feministas es pensar la forma de articular 
informaciones, estudios y activismos que puedan tomar en consideración las diferencias. Se trata de una parte importantísima de las discusiones a las que las redes y colectivos necesitan dedicarse, antes de que cualquier actividad hacia fuera pueda ser desarrollada. Sin embargo, muchas veces no es esa la realidad a la que los colectivos se enfrentan y los grupos se articulan con lo posible antes que con lo utópico.

Aunque la actividad entre músicas y feminismos, en Brasil, es desigual-como lo es el contexto del país-, consideramos que su presencia se disemina y se establece como estratégicas máquinas de guerra que hacen imposible que los pensamientos tradicionales sigan sin más cuestionamientos. Es de esa forma, como máquinas de guerra que constituyen experimentos para la práctica de la diferencia, que pensamos las prácticas alrededor de la música experimental, aunque ningún campo sea, en sí mismo, neutro de conflictos o unificado en sus objetivos.

\section{Grupo de Investigación en Estudios de Género, Cuerpo y Música (Universi- dad Federal de Rio Grande do Sul)}

El Grupo de Investigación en Estudios de Género, Cuerpo y Música ha sido creado en el 2013 como continuidad de los trabajos que venían siendo desarrollados desde el 2001 por el Grupo de Investigación en Musicología, los dos coordinados por Isabel Nogueira. Con el seguimiento de las investigaciones en musicología, el grupo ha enfatizado los estudios de género, pensando la creación sonora experimental como un campo posible, de experimentación de roles de género y desarrollo de la identidad artística. El grupo realiza cartografías de compositoras y creadoras de la escena musical brasileña y desarrolla actividades creadoras en el Colectivo Médula de Experimentos Sonoros, al lado del Grupo de Investigación en Creación Sonora y Grupo de Investigación Insubordinada, de Luciano Zanatta y Chico Machado, respectivamente.

\section{Feminaria Musical (Universidad Federal de Bahia)}

La Feminaria Musical es un grupo de investigación y experimentos sonoros feminista de extensión vinculado al Programa de Posgrado en Música de la Universidad Federal de Bahía (Brasil), que también integra la línea de investigación Género, Cultura y Arte del Núcleo de Estudios Interdisciplinarios sobre la Mujer (NEIM / UFBA), coordinado por Laila Rosa. Fundado en 2012, el grupo actualmente agrega profesores, profesoras y alumnado de diferentes sectores de la universidad. También incluye artistas colaboradores, que contribuyen de forma significativa para la investigación y para pensar nuevas propuestas, producción artística colectiva y militancia feminista, anti-racista y LGBT. El grupo realiza encuentros semanales para compartir experiencias, discutir la producción de conocimientos 
sobre canciones, feminismos postcoloniales, feminismo negro, relaciones étnico-raciales, la problematización de la blanquitud como categoría, sexualidades, teorías queer, cuestiones concernientes a la etnomusicología, performance, educación, música popular y estrategias de enfrentamiento a las matrices de desigualdades como el racismo, el etnocidio, el sexismo y la lesbo/homo/transfobia.

\section{Sonora Música y Feminismos}

Sonora Música y Feminismos es un grupo que surge en el 2015 debido a la necesidad de visibilizar y dialogar sobre el trabajo artístico de las mujeres. Como red colaborativa, reúne artistas y pesquisadores interesadas e interesados en manifestaciones feministas dentro del contexto de las artes. Propone la creación y ocupación de los espacios, la realización de investigaciones y debates, y la actuación con manifestaciones musicales de distintos tipos. Actualmente, Sonora Música y Feminismos realiza tres actividades regulares: un grupo de estudios con discusiones de textos y sesiones de escucha; la serie Voces, que recibe mujeres artistas para hablar sobre sus propios trabajos y la serie Visiones que recibe investigadores que se dedican a las áreas de género y feminismos. Este colectivo se encuentra atravesado por incertidumbres, indefiniciones, reticencias, aberturas, afectividades, sensibilidades y ruidos.

Aunque no pertenezca directamente a la Universidad de São Paulo, realiza sus reuniones en los espacios de esa universidad y muchos de sus participantes son o han sido estudiantes allí. Desarrollan actividades marcadamente en el Núcleo de Investigación en Sonología (NUSOM). Este núcleo tiene una actuación importante en la formación académica y artística en los estudios del sonido, al desenvolver actividades dentro y fuera del ambiente universitario. En el sitio web de Sonora se encuentra el registro de entrevistas con las compositoras/creadoras Janete el Haouli, Roseane Yampolschi, Valeria Bonafé, Ariane Stolfi y Barbara Biscaro. Algunos nombres del grupo que han estado en la creación de Sonora son, sin pretender ser exhaustivas, Lilian Campesato, Valeria Bonafé, Camila Zerbinatti, Flora Holderbaum, Tania Neiva, Isabel Nogueira, Eliana Monteiro da Silva, Natacha Maurer, Davi Donato.

\section{Sonora Ciclo Internacional de Compositoras}

Sonora Ciclo es un festival internacional de compositoras originado en Brasil en el 2016. Está organizado de forma colaborativa por autoras/productoras en distintas ciudades del mundo. Reconocemos que la presencia desigual de las mujeres en el mercado musical y la falta de representatividad pueden contribuir para desalentar a otras mujeres a emprender ciertos oficios en la música, tales como instrumentistas, regentes, arregladoras y compositoras. La incomodidad con ese contexto permitió que se formara una red de mujeres, en 
la cual la intersección entre ideas, disposición y gran emprendimiento de compositoras dio inicio a este festival. De acuerdo con las organizadoras del ciclo:

Trabajamos para que las mujeres en todo el mundo presenten con confianza sus sentimientos y opiniones a través de la música. A través de shows y otras actividades, el Festival Sonora constituye un lugar tanto de divulgación y exposición de las potencialidades individuales de las autoras, como también un espacio de reflexión colectiva, que promueve el encuentro de compositoras de varias vertientes y géneros musicales (Mussolini, 2017).

\section{Proyecto Disonantes}

Disonantes es una serie de conciertos de música experimental organizada por Renata Román y Natacha Maurer. Según las autoras, el proyecto surge en diciembre del 2015 como respuesta a la pregunta “¿dónde están las mujeres en la escena experimental?" provocada por la reducida presencia femenina en los conciertos que frecuentaban, sea como artistas, productoras o como público. Con el objetivo de modificar ese contexto y ampliar la participación de las mujeres en esa escena, la serie pretende crear un espacio de visibilidad e intercambio con performances de mujeres y/o grupos compuestos por un mínimo de $50 \%$ de mujeres que acontecen una vez al mes en espacios distintos en cada edición, en la ciudad de São Paulo, Brasil. Aunque no se trate de un colectivo estructurado, pensamos que la actuación del proyecto es una forma de activismo y contribuye a la reflexión sobre mujeres y música en la escena experimental. Además, fomenta las asociaciones entre mujeres para actuar en el proyecto, una vez que algunas de ellas siguen colaborando después de haber participado anteriormente.

\section{Colectivo Sopapo de Mujeres}

El Colectivo Sopapo de Mujeres es un colectivo feminista, autónomo, abierto y multidisciplinar que desarrolla proyectos dentro del tema mujer, arte y medios de comunicación. Trabaja directamente con el tema de la autoestima de mujeres negras, a partir del desarrollo de una investigación de sonidos de lo cotidiano, para la instauración de una zona de diálogo poético por medio de la creación de piezas sonoras y de entrevistas. Realiza el programa Ruidos Urbanos sobre radio arte y radio visual en un proceso integrado de formación continua en la frontera de distintos territorios de conocimiento, tales como arte, comunicación, política, feminismos, africanidades, desde el punto de vista práctico y teórico. Es reconocido como punto de medias libres por el Ministerio de la Cultura de Brasil. Clarissa Silveira, al hablar del punto de cultura, afirma que, así como en la vida, la transdisciplinariedad es una característica importante de las poéticas contemporáneas. El Colectivo Sopapo de Mujeres está formado por Clarissa Silveira, Cristina Nascimento, 
Denise Flores, Diane Barros, Janaina Dalla Vecchia y Marion dos Santos, y actúa en Porto Alegre, RS, Brasil.

Para hablar de las redes que contribuyen con el movimiento del campo de la música experimental, nos parece importante referir los blogs que acompañan y mueven las informaciones desde otros puntos de vista, del artista, o bien, del periodista o productor. Como serían muchos los ejemplos que tendríamos que referir para dar cuenta del campo, optamos por incluir tres ejemplos distintos, los blogs Volume Morto [Volumen Muerto], Filhas do Fogo [Hijas Del Fuego] y Dissonance from hell [Disonancia desde el infierno].

O Volume Morto: es un blog del periodista Gabriel "gg" Albuquerque que trata de músicas, sonidos, música experimental y todo lo que envuelve esas producciones, ya sea reseñas de discos, entrevistas o artículos de opinión. Mantiene el blog personal desde 2015, en el que entrevista a varios artistas de la escena experimental -en las que incluye a muchas mujeres- y escribe diversas entradas sobre el tema.

Filhas do fogo: es un blog de la periodista Amanda Cavalcanti que existe desde el 2016 y está dedicado a las mujeres de la música experimental en la ciudad de São Paulo. La periodista es asidua frecuentadora de la escena y entusiasta del aumento de la participación femenina en el campo. Colabora con la divulgación de esas artistas, escribiendo reportes y realizando entrevistas.

Dissonance from hell: es un blog, activo desde 2010, iniciado por el artista, compositor, productor, performer de Rio de Janeiro, Jhones Silva. En él, Silva presenta información de álbumes de música experimental lanzados, con énfasis en la música noise. También publica entrevistas y materiales de interés para la cultura de la música experimental y del ruido. El artista y productor es responsable por las colecciones Dissonance From Hell (dos volúmenes, 2012 y 2013) y los especiales Dissonance From Women (un volumen, 2014) y el Dissonance From Hell-América Latina (un volumen, 2016), además de otros discos de la escena de la música experimental.

Los blogs llaman la atención por el aporte a la formación de redes y diseminación de información vía internet, ya sea como páginas de autor o bien como colectivos que impulsan la reunión y actividad de compositoras, creadoras y productoras. Ejemplo importante de esa actividad es el grupo "Mujeres en la experimentación musical/Latinoamérica", creado por la artista sonora argentina Alma Laprida, que reúne un significativo número de creadoras brasileñas y de toda Latinoamérica. Alma dice, sencillamente, en la presentación: "este es un grupo para que empecemos a conocernos y a estar al tanto del trabajo de mujeres que experimentan con sonido en toda Latinoamérica. ¡Un abrazo!” (Laprida, s.f.). 
De esos encuentros, salieron las compilaciones Feminoise Latinoamérica, organizado por Maia Koenig; Tribute to Pauline Oliveros, organizado por Susan Campos Fonseca; Lego 10 Evas, organizado por Chip Musik Records y se está finalizando Mujeres Paisajistas Sonoras organizado por Ana María Romano Gómez, Vanessa Valencia Ramos y Fabian Esteban Luna. Aunque esas compilaciones no sean exclusivamente brasileñas, cuentan con creadoras brasileñas y son ejemplos de la generación de movimientos en red, en el que personas con distintos niveles de participación engendran un producto que no existía antes con ese formato.

\section{Festivales y encuentros}

Hablar de los festivales y encuentros que se celebran en Brasil es buscar estructurar una reflexión acerca de formas de red en sí misma y la visibilidad de esa producción. Por cierto, los festivales tienen su curaduría, sus objetivos y sus métodos de concepción, lo que buscaremos hacer es describir algunas iniciativas que no pretenden ser conclusivas y son, en su mayoría, provenientes de lo que los propios festivales divulgan, como parte de un trabajo de investigación en proceso. En el ámbito de los festivales y encuentros, damos una mirada a eventos que se realizan en la actualidad y que congregan muchas de las mujeres actuantes en el campo de la música experimental.

\section{Encuentro Nacional de Creatividad Sonora (ENCUN)}

El Encuentro Nacional de Creatividad Sonora (ENCUN) es un encuentro que reúne músicos y músicas, artistas que trabajan con sonido, investigadores e investigadoras y quienes más se interesan por el arte sonoro. Existe desde el 2003 y, en aquel momento, se llamaba Encuentro Nacional de Compositores Universitarios (ENCUN). Fue creado por un grupo de alumnos de composición de la Universidad de Campinas (UNICAMP), contaba con Valério Fiel da Costa, como líder del proceso al compositor. Desde su primera edición en 2003, el encuentro se caracteriza por no basarse en un proceso curatorial: mediante la inscripción, los compositores y compositoras envían sus obras y el festival las acoge, siempre que se tengan las condiciones estructurales para ello. Otra característica interesante del encuentro, que también demuestra una preocupación con respecto a la democratización y la representatividad, ahora en relación a la geopolítica, es su carácter itinerante. La participación de las mujeres aún es minoritaria, pero se incrementa a cada año: se podría decir que en la última edición ha crecido enormemente con relación a las anteriores (Neiva, Nogueira, Zerbinatti, Costa \& Zanatta, 2017). 


\section{Ibrasotope}

Ibrasotope es un colectivo/espacio/casa de producción, divulgación y promoción de música experimental en la ciudad de São Paulo. Nació en 2007 por iniciativa de los compositores Mário Del Nunzio y Henrique Iwao, que venían de los movimientos ocurridos en la UNICAMP y que dieron origen al ENCUN en 2003. Actualmente, el espacio es administrado por Mário Del Nunzio y por la artista Natacha Maurer, quien es la productora de Ibrasotope desde el 2010. Ibrasotope es una casa donde Del Nunzio es el morador más antiguo y por donde pasan o viven otros artistas en períodos determinados. Es, al mismo tiempo, un espacio cultural dedicado a la música experimental. Mantiene una agenda muy intensa que involucra conciertos, muestras, festivales y cursos; muchas veces con más de una actividad en la misma semana. Una de las actividades que puede ser considerada la columna vertebral del Ibrasotope es la "serie [IBR]", que son los conciertos de música experimental y contemporánea que ocurren en la casa. Esta serie existe desde la fundación y es, probablemente, la iniciativa más antigua con actividad permanente dedicada a la música experimental en la ciudad de São Paulo. De esta forma, promocionan, además de su propia agenda, alianzas con otras iniciativas, como el Proyecto Disonantes, el Improvise y otros. En 2015 ha sido creado, por ese colectivo, el Festival Internacional de Música Experimental (FIME), que ya contó con dos ediciones, en 2015 y 2016.

\section{Festival Internacional de Música Experimental (FIME)}

El Festival Internacional de Música Experimental (FIME), concebido y producido por Ibrasotope, tuvo su primera edición en julio de 2015 en la ciudad de São Paulo. El evento se pretende anual: su segunda edición aconteció en julio de 2016 y la tercera fue en noviembre del 2017. La programación del FIME envuelve conciertos, cursos y talleres de música experimental que ocurren en diversas localidades de la ciudad de São Paulo. En cada edición, la comisión organizadora propone un direccionamiento temático: en la primera edición, el tema ha fue "la idea de ruido, con su multiplicidad de interpretaciones y significados" (FIME, 2015). En la segunda edición, el tema explorado fue la idea de límite, la cual se explica en la convocatoria del evento y en el programa:

la idea de límite ... está abierta a la interpretación de los participantes, con sus múltiples acepciones y comprensiones. Algunas de ellas, presentes en la programación, se relacionan, por ejemplo: a los límites físicos del intérprete y del instrumento; a los límites entre música y ruido; a la interdependencia de los medios de comunicación; a los límites entre autoría y sus formas de reproducción; a la disolución de fronteras entre prácticas, géneros y formatos; entre otros (FIME, 2016).

ESCENA. Revista de las artes, 2018, Vol. 78, Núm. 1 (julio-diciembre), pp. 98-124. ISSN 2215-4906 


\section{Encuentro Internacional de Música y Arte Sonoro (EIMAS)}

El Encuentro Internacional de Música y Arte Sonoro (Eimas) es un festival gestionado por profesores universitarios y académicos, en la ciudad de Juiz de Fora, Minas Gerais. El sitio web del evento contiene información de las ediciones de los años 2012 y 2013. La primera de ellas organizada por Daniel Quaranta, Alexandre Fenerich y Luiz Eduardo Castelões, la segunda, organizada por Quaranta y Fenerich. No ha sido posible obtener de los organizadores mayor información de la que está en el sitio web, pero, creemos que en trabajos futuros eso será posible. Respecto a la presencia de mujeres en la edición del 2012, el encuentro tuvo la participación de las compositoras Sarah Cohen, Denise Garcia y Lilian Campesato. En la edición del 2013, las compositoras/creadoras participantes fueron Raquel Stolfi, Marion Velasco, Lilian Campesato, Lilian Nakahodo, Flora Holderbaum, Roseane Yampolschi, Thais Montanari y Laiana Oliveira.

\section{Kino Beat}

Kino Beat es un festival de música, performances audiovisuales, multimedia y artes integradas. A partir de los pilares imagen (Kino) y sonido (Beat), presenta artistas y actividades multidisciplinares, que utilizan alta y baja tecnología para la creación de sus trabajos. El arte experimental, sensorial, el concepto de inmersión, la búsqueda de nuevas posibilidades y conexiones artísticas son premisas importantes para la composición de su programa. Según su organizador y curador Gabriel Cevallos, el festival tiene por misión proponer experiencias, sorprender y desafiar, al mismo tiempo que trae, para sí, la labor de formar y consolidar un público para ese tipo de espectáculo. A la vez, actúa como agente estimulador para artistas locales y establece un punto de conexión con el mundo. Cevallos informa que el movimiento ha empezado en el 2009, con la organización de una muestra de películas relacionadas con la música. Luego de tres ediciones, en ese formato, ha surgido lo que Cevallos llamó "Kinobeat en vivo", como un embrión de lo que hoy es el Festival Kinobeat, que tiene lugar una vez al año, en la ciudad de Porto Alegre (RS) y que congrega artistas locales, nacionales e internacionales invitados

\section{Festival Novas Frequências (Nuevas Frecuencias)}

El Festival Nuevas Frecuencias es un festival que acontece en la ciudad de Rio de Janeiro desde el 2011, bajo curaduría de Chico Dub y Tathiana Lopes. Según sus curadores, el festival se preocupa por buscar artistas nuevos en la ciudad, en el área de la música experimental. La última edición, en diciembre de 2016, ha sido divulgada como el evento más importante de música experimental de América Latina y reunió representantes de trece países diferentes. 


\section{Perturbe}

Perturbe es un festival autodependiente organizado por el sello Meia-Vida en la ciudad de Curitiba desde 2012. Según los organizadores de la edición del 2015, el festival busca, en sus tres días de encuentro, "reunir expresiones políticamente y poéticamente productivas de ruido sonoro y performances artísticas" (Perturbe, 2015). El festival se caracteriza por abarcar propuestas que van desde performances abiertas, videos, cursos "do it yourself", hasta conciertos de música en el formato más tradicional. La idea principal difundida en el sitio del festival es de "estéticas marginales", según su edición del 2015. En este mismo año había, también, una indicación de incentivar prácticas y performances de ocupación de espacios no convencionales, abordando los procesos de creación:

en 2015 pretendemos abrir el evento para propuestas que desafíen la propia experiencia de crear o asistir a una performance, instalación o show, o bien que propongan una reflexión sobre sus condiciones de elaboración. Así, anhelamos cambiar la estructura del Perturbe para una nueva organización diferente de lo habitualmente visto en las producciones culturales y demás festivales (Perturbe, 2015).

\section{Festival Girls Rock Camp}

Girls Rock Camp Brasil es un campamento de vacaciones exclusivo para niñas de 7 a 17 años, que tiene como objetivo promover el empoderamiento de mujeres por medio de la música. Las actividades desarrolladas se dirigen al fortalecimiento de la autoestima, la desinhibición, el trabajo en grupo. Las niñas aprenden a tocar un instrumento, forman una banda, hacen una composición inédita y una presentación en vivo abierta a la comunidad. El proyecto existe en Brasil desde el 2013 y es pionero en América del Sur, forma parte de las Girls Rock Camp Alliance y hay alianza internacional del proyecto alrededor del mundo. El campamento Girls Rock Camp Brasil dialoga con el movimiento feminista desde el punto de vista de la interseccionalidad, ya que resulta importante la relación entre los recortes de género, raza, clase y sexualidad. Las feministas interseccionales perciben y enfatizan que las mujeres no sufren del mismo grado y forma de opresión, ya que las mujeres de diferentes realidades forman parte de otros sistemas que se refieren a cuestiones de raza, etnia, clase y sexualidad combinadas. De este modo, reconocen diferentes realidades además de las de mujeres de clase media, blancas y heterosexuales.

Aunque no sea específicamente volcado hacia la música experimental, su ubicación en el campo del rock and roll hace que se vincule al uso de la tecnología y de la cultura "do it yourself" (DIY), lo que muchas veces se convierte en un acercamiento al tema de lo experimental. Por otra parte, al tratar de los temas de educación de niñas y empoderamiento se relaciona, directamente, con las temáticas feministas y de los colectivos, que son objeto de 
ese trabajo. Algunas de las artistas vinculadas al Girls Rock Camp, pero con experiencia y actuación en la música experimental crean el sello "Hernia de Disco" y apoyan trabajos musicales artísticos experimentales de otras mujeres, por medio de álbumes y lanzamientos de trabajos en su plataforma. El sello está coordinado por las artistas: Brunella Martina, Desirée Marantes, Jiulian Regine (Duda), Liege Milk e Mariane Crestani. En sus propias palabras, son mujeres con experiencia en el underground, tanto de la música, como de otras artes, quienes se unen para abrir espacio a más proyectos integrados por mujeres en el escenario musical independiente.

\section{Consideraciones finales}

Hace pocos días, Facebook nos trajo la siguiente noticia:

el 12 de mayo de 2017, el Low Fi-Procesos Creativos promoverá, en asociación con el Flotar: Programa de Movilidad y Residencias Artísticas y apoyo del Goethe-Institut Salvador-Bahía (Brasil), iSe-lo! Netlabel y Mapê-Producción y Cultura, la segunda edición del proyecto Art Talks. La programación incluye rueda de conversación, charlas y performances artísticas, reuniendo artistas con trayectorias reconocidas en el circuito nacional/internacional. La iniciativa pretende promover un ambiente de intercambios en el que se discutirán estrategias de inserción de las mujeres en el contexto del Arte Electrónico / Experimental en Brasil, partiendo de las experiencias de cada invitada y con destaque para los eventos ya producidos por el Flotar en la Ciudad de México y Chicago. En el caso de las artistas sonoras, en una mesa llamada Género y Lenguajes, compuesta por la curadora Juci Reis (BA-MX), la artista visual y productora Andrea May (BA), además de las artistas sonoras Marina Mapurunga (CE), Barti Sena (CE) BA-UK) y Milena Kipfmüller (BR-ALE). En el caso de Renata Roman y Natacha Maurer (Colectivo Dissonantes-SP), Isabel Nogueira (RS), Marcela Lucatelli (BR-ITA) y la investigadora Tânia Neiva (PB), con interlocución de Edbrass Brasil, coordinador del proyecto (Art Talks, 2017).

Si bien, por una parte, esa noticia nos trae la idea de cómo es dinámico el campo, y cómo es imposible agotarlo en un artículo, trae también la referencia de que somos parte de ello y nuestra visión se forma desde dentro, en nuestro lugar específico como artistas, académicas, madres, mujeres blancas, de clase media, quienes vienen de una formación también académica en música. Creemos que pensar en música y feminismos es tener en cuenta este lugar de habla como un marcador de nuestro pensamiento, que no es innato, cristalizado, inmutable o estanque; pero que, al demarcarnos, busca deconstruir el concepto de neutralidad en música. Ese mismo concepto que naturalizó las practicas musicales como dependientes del patriarcado o inmersas en poder simbólico en lo que se refiere a repertorios y sus consideraciones sociales.

ESCENA. Revista de las artes, 2018, Vol. 78, Núm. 1 (julio-diciembre), pp. 98-124. ISSN 2215-4906 
Muchas personas, muchas mujeres producen en el ámbito de la música experimental en Brasil, nuestro esfuerzo ha sido mostrar que el campo es amplio e interceptado por prácticas, repertorios y significados provenientes de muchos lugares, y realizado por personas con distintas formaciones. Estamos seguras de que todavía mucho queda por hacer. Pensando en los proyectos y prácticas que listamos y de los que nos dedicamos a hablar aquí, creemos que pensar el feminismo va mucho más allá de buscar hacer una historia compensatoria, sino que se trata de imprimir nuevas formas de pensar, trabajar e investigar, generando mallas y texturas colaborativas donde las prácticas sean en sí mismas transformadoras.

Asimismo, cuestionar los saberes canónicos y hegemónicos, así como los sistemas de enseñanza y evaluación construidos según criterios que privilegian los contenidos por encima de las prácticas es una parte fundamental de una agenda feminista. En esos términos, buscamos hablar de grupos lo más diversos posibles, con diferentes formas de abordaje y diferentes conceptos del feminismo, algunos de una perspectiva artística o activista, otros con una mirada que viene del campo teórico, o bien, de una combinación de las dos. Ese es el primer resultado de un proyecto que está en curso y, en ese sentido, lo que presentamos aquí es un trabajo en proceso, con sus conceptos y formas de abordaje en constante delineamiento. Por el momento, estamos mapeando esas iniciativas y las mujeres que trabajan con música/sonido en Brasil.

A partir de ese primer muestreo de iniciativas, podemos percibir lo diverso y relativamente nuevo que es ese campo. En lo que concierne a la participación femenina, aunque no nos hemos inclinado en estadísticas, podemos percibir que muchas de las iniciativas que surgen con la temática de la representatividad, parten de la percepción de que los diversos campos de la música en el país son masculinizados; son protagonizados por hombres como productores, artistas y público. En el marco de esta constatación surgen iniciativas que cuestionan las razones de la falta de representatividad femenina. A la vez, trabajan en incentivar la participación de ese grupo, al promover, directa o indirectamente, empoderamiento femenino, mayor representatividad y, en un efecto cascada, otras mujeres pasan a participar y a interesarse por percibir la posibilidad de la ocupación de esos espacios y lugares por ellas/nosotras. El efecto cascada, también se extiende hacia los espacios y las iniciativas cuyo enfoque principal no necesariamente, pasa por la representatividad femenina o equidad de género, pues, tanto las mujeres se ven empoderadas para ocupar esos espacios, como los hombres, protagonistas de estos, pasan a percibirlas. Ellas/nosotras dejan/dejamos de ser invisibles y pasan/pasamos, aunque todavía, tímidamente, a ser consideradas como posibilidades de alianzas, de referencias y otros.

No es por casualidad que en el 2017, la tercera edición del Festival Internacional de Música Experimental (FIME) tuvo como meta alcanzar el 50 \% de participación de artistas 
mujeres y el $50 \%$ de participación de artistas hombres. Esa meta aún no ha sido conquistada en esa edición, pero se alcanzó aproximadamente, pues hubo un 47\% de participación femenina y el 53\%, masculina (FIME, 2017). Este porcentaje fue construido a lo largo de esos años en que el problema de la falta de representatividad o de equidad de género viene siendo pautado por las mujeres del campo. Así, reforzamos la importancia de iniciativas como Girls Rock Camp, la Red Sonora, el Sonora Ciclo, el Dissonantes en que el foco central son las mujeres.

Lo anterior no significa que todo funciona sin tensiones internas entre esos grupos y los más hegemónicos o que las iniciativas que buscan promover mayor representatividad o equidad son la respuesta para solucionar un problema mucho mayor a saber: el patriarcado y el capital. Ambos funcionan con la base de apropiación y explotación de grupos subalternos, siendo las mujeres (especialmente las minorías), los negros y las negras, los indígenas y las indígenas y todas las personas que no representan la cultura dominante y el agente dominante las mayores víctimas de apropiación y explotación.

En estas iniciativas, enumeradas aquí, vemos la posibilidad de que una pequeña semilla aparezca y muestre que existen otras formas de producción que pueden y son más inclusivas. ¿Quién sabe si tuviéramos cada vez más iniciativas que cuestionan y tensan el modus operandi (en que el hombre y todo lo que representa es la medida de las cosas), tendríamos otras referencias de formulación de pensamiento, arte, convivencia? Porque, en última instancia, no solo queremos ocupar un lugar. Queremos cambiar la forma con que ese lugar se construye, reproduce y consagra. Queremos cambiar los referenciales, para que no tenga más sentido pensar de forma binaria. Tal vez esas iniciativas, que traen algunas voces de la subalternidad a la superficie, sean un pequeño punto de luz en un cuarto oscuro

Al final de ese trabajo, traemos una cartografía inicial de mujeres que experimentan con el sonido, con sus nombres seguidos de sus enlaces de referencia. Para trabajos futuros, deseamos investigar las mujeres que trabajan con música experimental y son de una generación anterior a las que listamos aquí. Deseamos montar un sitio en línea, autogestionado con informaciones de compositoras, creadoras y productoras. Presentamos algunos retazos de una gran manta, que va tomando forma en la medida en que esos retazos se van configurando también. Así, entendemos que traerlos a la superficie, nos permite pensar en una colcha con diversos colores y formas. Con esos fragmentos del campo de la música experimental brasileña, hecha por mujeres, vamos cosiendo una colcha en donde las relaciones y conexiones entre esas mallas y fragmentos pueden surgir. En ese sentido, y también de forma experimental, entendemos que la construcción de esa narrativa se hace en el curso de nuestra investigación misma y, al mismo tiempo, buscamos traer una pluralidad de voces. Esas serán entretejidas junto con otros fragmentos, dándoles sentido y adquiriendo nuevos sentidos también. 
Con esto, traemos algunos vestigios de las actuaciones de mujeres en el campo de la música experimental brasileña, vestigios que contribuyen para conectar nuestro pensamiento y observar las texturas que se hacen y de qué forma actúan. Este ha sido nuestro propósito con esa reflexión, de forma experimental, seguras de que mucho queda por hacer.

\begin{tabular}{|c|c|c|}
\hline \multicolumn{3}{|c|}{ Cuadro 1. Listado de mujeres } \\
\hline Nome & Coletivo/projeto & Link \\
\hline Carina Levitan & & http://www.carinalevitan.com \\
\hline Flora Holderbaum & Editora da Revista Linda & $\begin{array}{l}\text { https://ufpr.academia.edu/FloraHolderbaum } \\
\text { http://lattes.cnpq.br/8864220630031373 }\end{array}$ \\
\hline Gabrielle Agah & & https://soundcloud.com/gabrielle-agah \\
\hline Isabel Nogueira & Medula/ Strana Lektiri & http://isabelnogueira.com.br/ \\
\hline Janete el Haouli & & https://pt-br.facebook.com/janete.el.haouli \\
\hline Leandra Lambert & Strana Lektiri/ Terra Incógnita & http://leandralambert.net \\
\hline Leticia Rita & & http://www.leticiarita.net \\
\hline Lilian Campesato & & http://liliancampesato.tumblr.com \\
\hline Lilian Nakao Nakahodo & & http://www.mapasonoro.com.br/ \\
\hline Lilian Zaremba & & $\begin{array}{l}\text { http://kunstradio.at/PROJECTS/CURATED_BY/ } \\
\text { ANEYE/index.html }\end{array}$ \\
\hline Luisa Nobrega & & http://www.luisanobrega.com \\
\hline Luisa Putterman & & http://www.luisa-puterman.com/ \\
\hline Luisa Toller & & https://www.cantodobrasil.com.br/luisatoller \\
\hline Marion Velasco & & $\begin{array}{l}\text { https://www.youtube.com/user/marionveloz ; } \\
\text { https://soundcloud.com/marion-velasco; http:// } \\
\text { empilhamento.blogspot.com.br/ }\end{array}$ \\
\hline Raquel Stolf & & http://www.raquelstolf.com \\
\hline Renata Roman & & http://renataroman.tumblr.com/ \\
\hline Vanessa de Michelis & & vnss.info \\
\hline Vivian Caccuri & & http://viviancaccuri.net/ \\
\hline Alice Grass & Nymphicus virus/Cerne & https://soundcloud.com/nymphicus-virus \\
\hline Grila que toca & $\begin{array}{l}\text { A banda que não existe/ } \\
\text { Cerne }\end{array}$ & \\
\hline
\end{tabular}

ESCENA. Revista de las artes, 2018, Vol. 78, Núm. 1 (julio-diciembre), pp. 98-124. ISSN 2215-4906 


\begin{tabular}{|c|c|c|}
\hline \multicolumn{3}{|c|}{ Cuadro 1. Listado de mujeres } \\
\hline Nome & Coletivo/projeto & Link \\
\hline Luisa Muccillo & Banku & $\begin{array}{l}\text { https://soundcloud.com/devirbanku ; https:// } \\
\text { soundcloud.com/luisa-muccillo }\end{array}$ \\
\hline Deborah Blank & The Bass Queen & https://soundcloud.com/deborah-blank9 \\
\hline Ana Paula Peroni & & https://soundcloud.com/ana-paula-peroni \\
\hline Vika Schmitz & & https://soundcloud.com/krunshe \\
\hline Bella & Meteoro & http://meteoro.hotglue.me/ \\
\hline Sannanda Acacia & Insignificanto & https://soundcloud.com/insignificanto \\
\hline Aline Vieira & $\begin{array}{l}\text { Flores feias/ Meia vida/Per- } \\
\text { turbe }\end{array}$ & https://meiavida.bandcamp.com/ \\
\hline Marina Mapurunga & & $\begin{array}{l}\text { www.sonatorio.org } \\
\text { https://ufrb.academia.edu/MarinaMapurunga } \\
\text { http://escritosdemar.tumblr.com/ }\end{array}$ \\
\hline Barti Sena & $\begin{array}{l}\text { http://cargocollective.com/ } \\
\text { bartira/BIO }\end{array}$ & $\begin{array}{l}\text { https://soundcloud.com/ill_will } \\
\text { http://bartholinsglands.tumblr.com/ } \\
\text { http://cargocollective.com/Abencoada } \\
\text { https://soundcloud.com/aben-oada666 }\end{array}$ \\
\hline Milena Kipfmuller & & http://www.milenakipf.de/pt \\
\hline Marcela Lucatelli & & $\begin{array}{l}\text { https://www.marcelalucatelli.co/ } \\
\text { https://soundcloud.com/marcela-lucatelli }\end{array}$ \\
\hline $\mathrm{Pa} \mathrm{Ma}$ & & http://cargocollective.com/1pa1 \\
\hline Skia - Saskia Peter & & https://soundcloud.com/skiapeter/ \\
\hline Erica Alves & & https://soundcloud.com/ericaalves \\
\hline Amanda Mussi & & https://soundcloud.com/ohmussi \\
\hline $\begin{array}{l}\text { Badsista- Rafaela } \\
\text { Andrade }\end{array}$ & & https://soundcloud.com/badsista \\
\hline Camila Alexandrini & Caosmose & https://caosmose.bandcamp.com/ \\
\hline Adriana PK & Caosmose & https://caosmose.bandcamp.com/ \\
\hline Lea Taragona & Dibuk & https://soundcloud.com/lea_cze \\
\hline
\end{tabular}




\begin{tabular}{|c|c|c|}
\hline \multicolumn{3}{|c|}{ Cuadro 1. Listado de mujeres } \\
\hline Nome & Coletivo/projeto & Link \\
\hline $\begin{array}{l}\text { Ruidos Urbanos Ra- } \\
\text { dioarte }\end{array}$ & $\begin{array}{l}\text { https://ruidosurbanospoa. } \\
\text { wordpress.com/ }\end{array}$ & https://soundcloud.com/ru-dos-urbanos \\
\hline Desiree Marantes & Harmonicos do Universo & https://soundcloud.com/mondevac \\
\hline Carla Boregas & Rakta & https://soundcloud.com/carlaboregas \\
\hline Claudia Holanda & & https://soundcloud.com/escutandoacidade \\
\hline alessa Camarinha & Editora da Revista Linda & https://soundcloud.com/alessamusic \\
\hline Bibiana Graeff & & https://soundcloud.com/bibianagraeff \\
\hline Natacha Maurer & & $\begin{array}{l}\text { https://soundcloud.com/natacha-maurer; http:// } \\
\text { natmaurer.tumblr.com/ }\end{array}$ \\
\hline Fernanda Aoki Navarro & & http://www.fernandanavarro.net/ \\
\hline Julia teles & Editora da Revista Linda & https://soundcloud.com/juliateles \\
\hline Thais Montanari & & http://www.thaismontanari.com/ \\
\hline Laiana Oliveira & & https://soundcloud.com/laiana-oliveira \\
\hline Jocy de Oliveira & & http://www.jocydeoliveira.com/ \\
\hline Carole Gubernikoff & & https://unirio.academia.edu/CaroleGubernikoff \\
\hline Denise Garcia & & $\begin{array}{l}\text { http://www.bv.fapesp.br/pt/pesquisador/4162/ } \\
\text { denise-hortencia-lopes-garcia/ }\end{array}$ \\
\hline Sarah Cohen & & $\begin{array}{l}\text { http://buscatextual.cnpq.br/buscatextual/visuali- } \\
\text { zacv.do?id=K4782897J2 }\end{array}$ \\
\hline Fuente: Elaboración pro & & \\
\hline
\end{tabular}

\section{Referencias}

Art Talks/SSA. (2017). Second edition/2017. [Página web]. Recuperado de http://art-talksssa.webnode.com/

Bourdieu, P. (2003). A Dominação Masculina. Rio De Janeiro: Bertrand Brasil.

Bourdieu, P. (2004). A Economia Das Trocas Simbólicas. São Paulo: Perspectiva.

Campesato, L. (2015). Discursos e Ideologias do ‘experimentalismo’ na música do pós-guerra. Revista Poiésis, 25, 43-64. Recuperado de http://www.poiesis.uff.br/p25/p25-dossie-3-lilian-campesato.pdf 
Del Nunzio, M. (2017). Práticas Colaborativas Em Música Experimental No Brasil Entre 2000 E 2016 (tesis de doctorado). Universidad de São Paulo, São Paulo, Brasil. Recuperado de http://www.teses.usp.br/teses/disponiveis/27/27158/tde-05092017-095356/pt-br. php, file:///C:/Users/Usuario/Downloads/MarioAugustoOssentDelNunzio.pdf

Encun X. (2012). Programa Do Encontro De Compositores Universitários-Encun X 2012. Realizado No Rio De Janeiro Rj [blog]. Recuperado de https://encun2012.wordpress.com/

Encun XI. (2013). Programa Do Encontro De Compositores Universitários-Encun 2013. Realizado en João Pessoa, Pb [blog]. Recuperado de https://encun2013.wordpress.com/

Encun XII. (2014). Programa Do Encontro De Compositores Universitários-Encun 2014. Realizado em São Paulo, SP Recuperado de http://www.ibrasotope.com.br/2014/10/ de-31-de-outubro-09-de-novembro-o-xii.html

Encun XIII. (2015). Programa Do Encontro De Compositores Universitários-Encun 2015. Realizado en Campinas, SP [blog]. Recuperado de http://encun.nics.unicamp.br/

Festival Girls Rock Camp. (s.f.). Site Oficial do Festival [página web]. Recuperado de http:// grcportoalegre.com/

Festival Internacional de Música Experimental I, FIME. (2015). Programa Do Primeiro Festival Internacional De Música Experimental-Fime I. São Paulo [página web]. Recuperado de: http://www.fime.art.br/2015/pt/

Festival Internacional de Música Experimental II, FIME II. (2016). Programa Do 2o Festival Internacional De Música Experimental-Fime II. São Paulo [página web]. Recuperado de: http://www.fime.art.br/2016/pb/

Festival Internacional de Música Experimental III, FIME III. (2017). Programa do 3o Festival Internacional de Música Experimental-Fime III. São Paulo [página web]. Recuperado de http://www.fime.art.br/2017/sobre/

Filhas do Fogo. (s.f.). O sintético e o orgânico no trabalho de anelena toku [blog]. Recuperado de http://filhasdofogo.tumblr.com/

Fonseca, S. \& Nogueira, I.P. (coordinadoras). (2013). Estudos De Gênero, Corpo E MúsicaAbordagens Metodológicas (Vol. 3). Porto Alegre: Anppom.

Gelain, G. \& Nogueira, I. (2017). Girls Rock Camp promove empoderamento feminino através da música em sua edição de Porto Alegre. São Paulo: Women's Music Event [página web]. Recuperado de http://womensmusicevent.com.br/girls-rock-camp-promove-empoderamento-feminino-atraves-da-musica-em-sua-edicao-de-porto-alegre/ 
Hooks, B. (2013). Ensinando A Transgredir. A Educação Como Prática Da Liberdade. (Trad. Marcelo Brandão Cipolla). São Paulo: Martins Fontes.

lazzetta, F. (2014). Entre A Pesquisa E A Criação: A Experiência Dentro Da Sonologia. Anais Do XXIV Congresso Da Associação Nacional De Pesquisa E Pós-Graduação Em Música, São Paulo. 1-9. Recuperado de http://www.anppom.com.br/congressos/index. php/24anppom/SaoPaulo2014/paper/view/3014/763

Ibrasotope. (s.f.). Programas Do Ibrasotope De 2007-Ago/2016 [página web]. Recuperado de http://www.ibrasotope.com.br/

Iwao, H. \& Nunzio Del, M. (coordinadores). (2009). Ibrasotope, Revista De Música Experimental. Recuperado de http://henriqueiwao.seminalrecords.org/arquivos/pdf/2009_ del_nunzio-iwao-ibrasotope_um_ano.pdf

Iwao, H. (2012). Colagem Musical Na Música Eletrônica Experimental. [Tesis de maestría] Programa de Posgrado en Música, Escola De Comunicação e Artes da Universidade de São Paulo - USP, São Paulo, Brasil.

Laprida, A. (s.f.) Mujeres en la experimentación sonora/ Latinoamérica [grupo de Facebook]. Recuperado de https://www.facebook.com/groups/1622622487999222/

Lucatelli, M. (2016). Sobre Dissonantes E Wesley Safadão. Linda Revista sobre cultura electroacústica, 3(3), Recuperado de http://linda.nmelindo.com/2016/05/ sobre-dissonantes-e-wesley-safadao/

Mohanty, C. T. (1984). Under Western Eyes: Feminist Scholarship and Colonial Discourses. Boundary 2, 12(3), pp. 333-358. Recuperado de http://www.jstor.org/stable/302821

Mussolini, D. (2017). A Revolução Virá Pelo Ventre [página web]. Recuperado de https:// www.sonorafestival.com/

Neiva, T.M., Nogueira, I., Zerbinatti, C.D. Costa, V.F. \& Zanatta, L. (2017). Sobre o Encun: Textos a Dez Mãos e Cinco Cabeças. Linda. Revista sobre cultura elctroacústica, 4(1). Recuperado de http://inda.nmelindo.com/2017/02/ sobre-o-encun-texto-a-dez-maos-e-cinco-cabecas/

Nogueira, I. \& Zanatta, L. (2016). Impermanente Movimento: Epistemologias Feministas, Tecnologias, Performance E Criação Sonora Em Loops E Camadas De Sentido. Revista Sonic Ideas. En prensa.

Palombini, C. (2003). Música Lésbica E Guei, De Philip Brett \& Elizabeth Wood: Apontamentos de Tradução. Per Musi-Revista De Performance Musical, 8, 157-164. Recuperado 
de http://www.musica.ufmg.br/permusi/port/numeros/08/num08_resenha1.pdf

Perturbe-Festival. (2015). Página de divulgação do festival [página web]. Recuperado de https://Meiavida.Hotglue.Me/Sobre

Possas, L. (2011). As Fronteiras: Retomando A Palavra E Libertando Significados. Quem Sou Eu? As Mulheres E As Identidades Redescobertas. Revista Territórios E Fronteiras, 4(1), 59-70.

Sonora. (s.f.). Site Da Rede Sonora-Músicas E Feminismos [página web]. Recuperado de http://www.sonora.me/

Sopapo De Mulheres. (s.f.). Site Do Coletivo [página web]. Recuperado de http://Quilombodosopapo.Redelivre.Org.Br/Sopapo-De-Mulheres/

Travassos, E. (2005) Pontos de escuta da música popular no Brasil. En E. Travasso y M. Ulhoa (Eds.), Música popular na América Latina: pontos de escuta. Porto Alegre: Editora da UFRGS.

Zerbinatti, C.; Nogueira, I. y Pedro, J. (2017). A Emergência do Campo de Música e Gênero no Brasil: um tecido de malhas híbridas a partir de reflexões das musicologias e epistemologias feministas. Descentrada. Revista Interdisciplinaria de Feminismos y Genero. En prensa. 